\title{
Intracerebral haemorrhage: a model for the prediction of outcome
}

\author{
RUSSELL K PORTENOY,* RICHARD B LIPTON,* ALAN R BERGER,* \\ MARTIN L LESSER, $\uparrow$ GEORGELANTOS,$\ddagger$
}

From the Departments of Neurology, ${ }^{*}$ Biostatistics, $\dagger$ and Radiology, $\ddagger$ the Albert Einstein College of Medicine, New York, USA

SUMmARY The hospital charts and CT scans of 112 patients with spontaneous supratentorial haemorrhage were retrospectively reviewed to evaluate factors important to prognosis. A low Glasgow Coma Scale score, coma, ataxic respiration, abnormal pupils, acute hypertension, large haemorrhage size and intraventricular extension of blood were associated with a poor outcome. Multivariate analysis using the technique of logistic regression identified three variables, the Glasgow Coma Scale score, haemorrhage size and intraventricular extension of blood, which were most predictive of outcome. A model was developed from this analysis which accurately estimates the probability of good outcome and the risk of poor outcome based on data available on presentation. With additional validation, this model may be useful in choosing patients for surgical evacuation of haematomas.

The advent of computed tomography (CT) has permitted earlier and more accurate diagnosis of supratentorial intracerebral haemorrhage (ICH). ${ }^{1}$ This has led to the recognition that both presentation and prognosis in ICH vary widely. ${ }^{2-6}$ These two factors (early diagnosis by $\mathrm{CT}$ and the variability of outcome) have fuelled a controversy over the efficacy of surgical evacuation in acute $\mathrm{ICH} .^{78}$ Though individual patients appear to benefit, there is as yet no welldefined population whose outcome consistently improves with surgery. The accurate determination of prognosis in medically treated ICH is an essential, and still elusive, first step in defining the patient population likely to benefit from surgery.

Predictors of outcome in ICH have been evaluated in numerous studies. ${ }^{24689-15}$ All have limited applicability due to the use of univariate analysis, which fails to account for interactions among prognostic factors, or to the dependence on sophisticated CT analytical techniques unavailable to the clinician. We have developed an easily applied and accurate prognostic model based on a multivariate analysis using the statistical technique of stepwise logistic re-

Address for reprint requests: Dr $\mathbf{R}$ Portenoy, Department of Neurology, Albert Einstein College of Medicine, 1300 Morris Park Avenue, Bronx, New York 10461, USA.

Received 31 January 1986 and in revised form 9 May 1986. Accepted 15 May 1986 gression. With additional validation, this model may be a useful first step in the selection of appropriate treatment based on the prognosis.

\section{Methods}

The hospital charts and CT scans of all patients admitted to the Bronx Municipal Hospital Center of the Albert Einstein College of Medicine with the diagnosis of non-traumatic, non-neoplastic, supratentorial $\mathbf{I C H}$ during the years 1981-1983 were evaluated. Diseases potentially related to ICH were noted, including chronic hypertension, bleeding diathesis (including use of anticoagulants), atrial fibrillation, prior stroke, dementia, vasculitis, and aneurysm or arteriovenous malformation (AVM) (known previously or later documented angiographically or at necropsy). All patients were evaluated by a neurologist on admission to the emergency room. In all cases, patients were assessed within hours of their ictus. The following clinical features were recorded from this examination: respiratory abnormality or blood pressure elevation; the occurrence of headache, vomiting or seizures; level of consciousness (alert, lethargic, stuporous or comatose); aphasia or other discrete deficit in higher cortical function; and abnormalities in pupillary function, gaze, visual fields, corneal reflexes, and motor or sensory function. From this evaluation, a Glasgow Coma Scale (GCS) score ${ }^{16}$ was computed.

All patients had CT scans within several hours of the evaluation by the neurologist. These were evaluated by at least two of the authors without prior knowledge of patient outcome. ICH location, size, and the presence of midline shift, hydrocephalus, or extension of blood into the ventricles or 
subarachnoid space were recorded. Though some studies of ICH eliminate patients with aneurysm, AVM, bleeding diathesis or a high risk of cardiac emboli, these criteria vary among authors. The present study excluded only patients with bleeding into a neoplasm, since the tumour itself may alter presentation and outcome, and patients with haemorrhagic infarct on CT, using the radiographic criteria described by Kase et al. ${ }^{17}$ Extension of blood into any ventricle was noted and the degree of spread into the lateral ventricles was recorded as none, mild $(<10 \%)$, moderate $(10-50 \%)$, or severe $(>50 \%)$. Blood in the subarachnoid space was noted as either present or absent. Hydrocephalus was defined as enlargement of the lateral ventricles without concurrent expansion of the cisternal or sulcal spaces. The location of the haemorrhage was defined as lobar if it appeared in the subcortical white matter, wholly outside the basal ganglia and thalamus; haemorrhages contacting these structures were labelled as deep. An index of haemorrhage size was computed as the average of the largest diameter of the ICH and the midline perpendicular of this line in the CT plane with the greatest area of haemorrhage, divided by the biparietal diameter measured at the high ventricular cut. Though this index only roughly approximates haemorrhage volume, its strong association with outcome (see below) supports its validity. In addition, it can be determined by the clinician at the time of admission without sophisticated software, and permits comparisons among patients studied on different CT scanners by indexing ICH size to biparietal diameter. For purposes of data analysis, ICH size was divided into quartiles.

Outcome was graded as no deficit, mild deficit, moderate deficit (impaired but functionally independent), severe deficit (dependent on others for activities of daily living), persistent vegetative state, and death. For most analyses, patients were divided into a good outcome group who were functionally independent (first three categories) and a poor outcome group who were functionally dependent or worse (last three). For those patients who survived beyond 2 weeks, outcome was determined both one to 2 weeks after admission and again at last recorded follow-up. For most patients, the last recorded follow up comprised death or discharge after the acute hospitalisation. A small number of patients were followed in the outpatient department after discharge and the last recorded follow up was obtained from the assessment of the clinic physician. Analysis of these data revealed that only four patients moved between good and poor outcomes from the early to the late determinations. All outcome analyses were therefore done using information from the last recorded evaluation. It should be recognised that this interval for outcome determination is arbitrary, that prognostic indicators can be derived for outcome at any point in time. Though the last recorded follow up was the most relevant to long-term prognosis in the present study, it does not exclude the possibility that outcomes may have continued to change in the minority of patients who were discharged after their acute hospitalisation and were then lost to follow up. The significance of these late changes is doubtful, since most of those with poor outcome died and all of those with good outcome were already functionally independent.

The data were first analysed in a univariate fashion using either the chi-square or Fisher exact test. Multivariate anal-
Table 1 Outcome in intracerebral haemorrhage

\begin{tabular}{|c|c|c|c|}
\hline & $\begin{array}{l}\text { Good } \\
\text { outcome } \\
(\%)\end{array}$ & $\begin{array}{l}\text { Poor } \\
\text { outcome } \\
(\%)\end{array}$ & $p$ value \\
\hline GCS Score: $\begin{aligned} 3-4(N=30) \\
5-8(N=22) \\
9-13(N=27) \\
14-15(N=33)\end{aligned}$ & $\begin{aligned} & 1(3) \\
& 1(5) \\
& 11(41) \\
& 28(85)\end{aligned}$ & $\begin{aligned} 29 & (97) \\
21 & (95) \\
16 & (59) \\
5 & (15)\end{aligned}$ & $<0.001$ \\
\hline Alertness $(\mathrm{N}=28)$ & $24(86)$ & 4 (14) & $<0.001$ \\
\hline Coma $(\mathrm{N}=48)$ & $1(2)$ & 47 (98) & $<0.001$ \\
\hline Ataxic respirations $(\mathrm{N}=25)$ & $0(0)$ & $25(100)$ & $<0.001$ \\
\hline Abnormal pupils $(\mathrm{N}=45)$ & 2 (4) & $43(96)$ & $\begin{array}{l}<0.001 \\
<0.05\end{array}$ \\
\hline Haemorrhage size: & & & \\
\hline $\begin{array}{r}4-19(\mathrm{~N}=29) \\
20-28(\mathrm{~N}=28) \\
29-44(\mathrm{~N}=30) \\
45-81(\mathrm{~N}=25)\end{array}$ & $\begin{array}{r}21(72) \\
14(50) \\
5(17) \\
1(4)\end{array}$ & $\begin{aligned} 8 & (28) \\
14 & (50) \\
25 & (83) \\
24 & (96)\end{aligned}$ & $<0.001$ \\
\hline $\begin{array}{l}\text { Intraventricular spread } \\
(\mathbf{N}=69)\end{array}$ & $12(17)$ & $57(83)$ & $<0.001$ \\
\hline
\end{tabular}

Good outcome is defined as no worse than moderate functional impairment, while poor outcome includes no independent function, vegetative state, coma and death. Haemorrhage size, divided into quartiles, is defined by a calculated index (see text).

ysis, using stepwise multiple logistic regression, ${ }^{18}$ was then done to determine which factors were simultaneously prognostic for outcome as defined above. Dummy variables were used to represent nominal scale variables. The adequacy of the fitted models were tested using the goodness-of-fit test. All statistical results were declared significant if $\mathrm{p}<0.05$.

\section{Results}

Fifty two patients (46\%) with ICH died before day 14 following their ictus. Of those alive at 2 weeks, median follow up was 60 days (range 15-790 days).

Forty one patients $(37 \%)$ were functionally independent at last follow up, while outcome was poor in $71(63 \%)$ patients, including $65(58 \%)$ who died. Poor outcome was significantly associated with the following features at presentation: coma, ataxic respiration, pupillary abnormality, acute HTN, IV spread of blood, the GCS score and large ICH size (table 1). An analysis of patients with IV extension revealed that the probability of poor outcome increased with the estimated volume of IV blood. GCS score was particularly predictive when 8 or below; greater than $95 \%$ of these patients had a poor outcome. The index of ICH size ranged from 0.04 to 0.81 and was also a highly significant factor in the univariate analysis; $89 \%$ of patients with greater than the median ICH size did poorly, while $38 \%$ with smaller than median ICH had a poor outcome.

ICH location did not significantly influence outcome. Nineteen $(45 \%)$ of patients with lobar and 26 $(37 \%)$ of those with deep haemorrhages had a favourable outcome. Location, however, interacted with ICH size, suggesting that haemorrhage location may have impact in certain settings. No difference in outcome was found in both the smallest and largest size quartiles; more than $70 \%$ of patients with either lobar 
Table 2 Results of multivariate logistic regression analysis to estimate the probability of good outcome.

\begin{tabular}{llll}
\hline Variable & Coefficient & Standard error & $p$ value \\
\hline Intercept & -1.715 & 1.409 & NS \\
GCS sum & 0.389 & 0.095 & $<0.001$ \\
ICH size & -0.068 & 0.025 & $<0.007$ \\
IV spread & -1.649 & 0.645 & $<0.011$ \\
\hline
\end{tabular}

Probability (good outcome) $=1 /(1+\operatorname{Exp}(+1 \cdot 715-0.389$ GCS sum +0.068 ICH size +1.649 IV spread)).

GCS sum = sum of best motor, best verbal, best eye opening subscale scores.

ICH size, defined by a calculated index (see text), represents grouped values based on size quartile, as follows:

$\mathrm{ICH}$ size $=14$ if size $=0.04-0.19$

$$
\begin{aligned}
& =24 \text { if size }=0 \cdot 20-0.28 \\
& =36 \text { if size }=0 \cdot 29-0.44 \\
& =60 \text { if size }=0 \cdot 45-0.81 .
\end{aligned}
$$

IV spread $=1$ if IV spread is present, 0 if not.

or deep ICH did well and only one patient with lobar ICH and none with deep haemorrhage had a favourable outcome in the group with largest size. In the middle two size quartiles, however, a good outcome
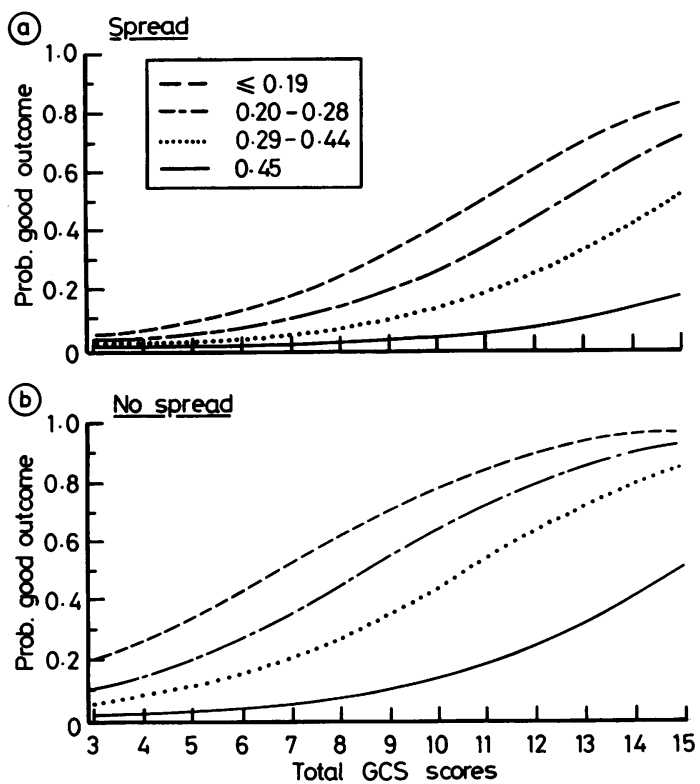

Fig Estimated probability of a good outcome in $\mathrm{ICH}$. Each curve is identified by the presence $(a)$ or absence $(b)$ of intraventricular spread and by a particular size quartile. The estimated probability of a good outcome is read off the ordinate after selecting the Figure which categorises intraventricular spread and the curve which indicates the computed ICH size index, then moving along the curve to the point on the abscissa representing the correct GCS score. For example, a patient whose ICH has no intraventricular spread, is between 0.20 and 0.28 calculated units in size (see text), and whose admission GCS score is 15 has an estimated probability of a good outcome of approximately 0.92. occurred in $46 \%$ with lobar and only $21 \%$ with deep ICH.

Outcome was also unaffected by method of treatment. Of sixteen patients treated by craniotomy, outcome was good in $6(38 \%)$ and poor in $10(62 \%)$, proportions almost identical to those in the larger group treated medically ( $36 \%$ vs $64 \%$, respectively). Since this survey could not of course control for the variable selection criteria used in choosing patients for surgery, these results cannot be used to evaluate the efficacy of this type of treatment; rather, these data suggest only that surgery was not an important factor in determining prognosis in this population.

Interactions among these factors were further defined with a multivariate analysis using stepwise logistic regression. This revealed that the combination of three variables, GCS score, ICH size, and IV extension of blood, were significantly associated with good outcome (table 2). A model was constructed from this analysis which allows an estimation of the probability of good or poor outcome (fig). Using this model, $88 \%$ of patients with poor outcomes and $87 \%$ of patients with good outcomes were correctly identified from information available on admission.

\section{Discussion}

Though outcome in ICH has been evaluated in several studies, detailed statistical analysis has rarely been attempted. In previous surveys, overall mortality has ranged from $14-50 \%,{ }^{1468-10} 19$ with functional recovery in somewhat less than half all patients. In the present study, $58 \%$ of patients died and a good functional recovery was attained by $37 \%$. The level of function at 2 weeks after the ictus was maintained in almost every case at the last recorded evaluation.

Of all factors evaluated, coma $468-12151720$ and large ICH size 48-1014151720 have most consistently been associated with poor prognosis, though even the latter has been found to be unrelated to outcome in one study. ${ }^{6}$ Extension of blood into the IV ventricle has also usually, ${ }^{12610141721}$ though not invariably, ${ }^{11}$ related to poor outcome and the severity of intraventricular extension was identified as an important factor in one study, ${ }^{22}$ a finding confirmed in the present report. Other series have suggested that site of bleeding in the basal ganglia ${ }^{8}$ and the findings of gaze palsy, ${ }^{4}$ bilateral plantar extensor responses, ${ }^{11}$ and chronic $\mathrm{HTN}^{17}$ predict poor outcome. Finally, several studies have concluded that patients with lobar ICH have a better prognosis than those with deep $\mathrm{ICH},{ }^{4612151719}$ while others have found no association. ${ }^{2} 1014$

In the initial, univariate analysis of our data, level of consciousness, ICH size and intraventricular extension were also associated with outcome. In addition, 
the presence of ataxic respirations, abnormal pupillary responses and HTN at the time of presentation were prognostically important. The computed GCS score, ${ }^{16}$ which has only rarely been used in the evaluation of non-traumatic $\mathrm{ICH},{ }^{22}$ was also found to be highly associated with outcome. This analysis, however, like the others cited above, did not assess the interaction of these factors or provide a prognostic model capable of generating the relative risk of a poor outcome based on their combination.

Such a model was produced by a multivariate statistical analysis using stepwise logistic regression. This method established that the most important factors evaluated as potential prognostic indicators in our population were the GCS score on presentation, ICH size and intraventricular extension of blood. From the model developed by this analysis, the probability of a good or poor outcome can be estimated with a high degree of sensitivity and specificity (fig 1 ).

Though this model must be applied to an independent sample of patients to further validate and refine it, it appears to offer an accurate analysis of risk based on data available on presentation. This information may assist in clinical decision-making and in advising patients or family about prognosis. Ultimately, rational decisions regarding surgical treatment should be based on the known risks of the procedure, potential benefits, and the prognosis with medical treatment alone. In addition, the factors identified by this analysis should be controlled in future studies of therapy in ICH. To avoid bias, patient groups should be matched for combinations of these factors and the success of randomisation assessed by determining whether different patient groups were equivalent in prognosis prior to the intervention.

\section{References}

1 Weisberg LA. Computerized tomography in intracranial hemorrhage. Arch Neurol 1979;36:422-6.

2 Douglas MA, Haerer AF. Long-term prognosis of hypertensive intracerebral hemorrhage. Stroke 1982; 13:488-91.

3 Feindel W. Management of intracerebral hemorrhage. In: Goldstein M, et al, eds. Advances in Neurology, vol 25. New York: Raven Press, 1979:293-307.

4 Helweg-Larsen S, Sommer W, Strange P, Lester J, Boysen G. Prognosis for patients treated conservatively for spontaneous intracerebral hematomas. Stroke 1984;15:1045-8.

5 Ropper AH, Davis KR. Lobar cerebral hemorrhages: acute clinical syndromes in 26 cases. Ann Neurol 1980;8:141-7.

6 Steiner I, Gomori JM, Melamed E. The prognositic value of the CT scan in conservatively treated patients with intracerebral hematoma. Stroke 1984;15:279-82.

7 Masdeu JC, Rubino FA. Management of lobar intracerebral hemorrhage: medical or surgical. Neurology 1984;34:381-3.

8 Waga S, Yamamoto Y. Hypertensive putaminal hemorrhage: treatment and results. Stroke 1983;14:480-4.

9 Bolander HG, Kourtopoulos H, Liliequist B, Wittboldt S. Treatment of spontaneous intracerebral hemorrhage: a retrospective analysis of 74 consecutive cases with special reference to computer tomographic data. Acta Neurochir 1983;67:19-28.

10 Garde A, Bohmer G, Selden B, Neiman J. 100 cases of spontaneous intracerebral haematoma. Eur Neurol 1983;22:161-72.

11 Kwak R, Kadoya S, Suzuki T. Factors affecting the prognosis in thalamic hemorrhage. Stroke 1983; 14:493-500.

12 Paillas JE, Alliez B. Surgical treatment of spontaneous intracerebral hemorrhage. Neurosurgery 1973;39: 145-51.

13 Pertuiset B, Sichez JP, Yacoubi A, Gardeur D, Melon E, Haddad K. 96 cases of spontaneous medical cerebral hemorrhage: diagnostic therapeutic experience. Rev Neurol (Paris) 1983;139(5):359-66.

14 Stein RW, Caplan LR, Hier DB. Outcome of intracranial hemorrhage: role of blood pressure and location and size of lesions. Ann Neurol 1983;14:132.

15 Volpin L, Cervellini P, Colombo F, Zanusso M, Benedetti A. Spontaneous intracerebral hematomas: a new proposal about the usefulness and limits of surgical treatment. Neurosurgery 1984;15:663-6.

16 Jennett B, Teasdale G. Management of Head Injuries. Philadelphia: FA Davis Co, 1981:77-84.

17 Kase CS, Williams JP, Wyatt DA, Mohr JP. Lobar intracerebral hematomas: clinical and CT analysis of 22 cases. Neurology 1982;32:1146-50.

18 Lee ET. Statistical Methods for Survival Data Analysis. Belmont, CA: Lifetime Learning Publications, 1981.

19 Mohr JP, Caplan LR, Melski JW, et al. Clinical findings in intracerebral hemorrhage from the Harvard Stroke Registry. Neurology 1976;26:381-6.

20 Barraquer-Bordas L, Illa I, Escartin A, Ruscalleda J, Marti-Vilalta JL. Thalamic hemorrhage: a study of 23 patients with diagnosis by computed tomography. Stroke 1981;12:524-7.

21 Wiggins WS, Moody JF, Toole JF, Laster DW, Ball MR. Clinical and computerized tomographic study of hypertensive intracerebral hemorrhage. Arch Neurol 1978;35:832-3.

22 de Weerd AW. The prognosis of intraventricular hemorrhage. J Neurol 1979;222:45-51. 